\section{Interleukin-1 and the Treatment of Auto-inflammatory Diseases}

\author{
C. A. Dinarello ${ }^{1}$ \\ 1 University of Colorado Denver, Aurora, Colorado, USA
}

Chronic inflammatory diseases fall into two categories: either "autoimmune" or "auto-inflammatory" [1]. Although nearly all autoimmune diseases have an inflammatory component, as in rheumatoid arthritis, in autoimmune diseases, the primary defect is the auto-reactive T-cell. The term "auto-inflammatory diseases" encompasses several local and systemic diseases due to monocyte dysfunction, each responsive to blocking interleukin-beta (IL-1 $\beta$ ) or blocking the IL-1 receptor using the IL-1 receptor antagonist. The best example is Familial Mediterranean Fever since manifestations of this disease include fever, fatigue, painful inflamed serosal and synovial tissues, biochemical markers of systemic inflammation, and hematological responses of neutrophilia [2]. Other auto-inflammatory diseases are due to single amino acid mutations in the protein "cryopyrin" [3] and are called "cryopyrinopathies"; the same syndromes are also termed "cryopyrin associated periodic syndromes" (CAPS). Systemic inflammation can be lethal, and the best example is septic shock. However, septic shock represents exogenously induced inflammation or simply "exogenous inflammation." Although many laboratory studies use bacterial products to stimulate IL- $1 \beta$ production, in the clinical sense, auto-inflammation is the result of IL-1-induced IL-1 or IL-1 induction of other cytokines such as IL-17. Either IL- $1 \alpha$ or IL-1 $\beta$ can be the driving component in auto-inflammation. For example, in patients with neonatal onset multi-inflammatory disease (NOMID) treatment with the IL-1 receptor antagonist anakinra drives down not only the manifestations of the disease but also gene expression for IL- $1 \alpha$, IL-1 $\beta$, TNF $\alpha$, IL- 6 and caspase- 1 [4]. The most compelling evidence for the importance of IL- 1 in inflammation comes from persons born with a mutation in the gene that codes for the IL-1 receptor antagonist. The syndrome is called DIRA for deficiency of the IL-1 receptor antagonist $[5,6]$. At birth, these patients develop lethal inflammation with massive numbers of neutrophils in the skin and high levels of IL17.

Although traditionally "auto-inflammatory diseases" are thought to be examples of rare diseases, in reality several common diseases such as type 2 diabetes, gout, dry eye disease, hydradenitis suppurativa, and heart failure are likely to be auto-inflammatory conditions. The common link is loss of the tight control over the processing and secretion of IL- $1 \beta$ from the activated monocyte. The processing and secretion of IL- $1 \beta$ is a function of the IL-1 $1 \beta /$ caspase- 1 "inflammasome", a complex of intracellular proteins that results in the secretion of IL-1ß. IL-1 receptor blockade or neutralization results in a rapid and sustained reduction in disease severity; the responses are highly consistent with blockage of IL- $1 \beta$ activity in these diseases. The measurement of IL- 6 in the circulation is the preferred method for assessing the severity of the inflammation in auto-inflammatory diseases, and the fall in se- rum IL-6 reveals the effectiveness of anti-IL-1 treatment. In cytokine biology, auto-inflammatory diseases are examples that reveal the causative role of a specific cytokine for disease severity. In addition to treating CAPS with IL-1 blockade, several trials are currently underway, including type 1 and prediabetes, post-myocardial infarction heart failure, osteoarthritis, gout and smoldering myeloma. CANTOS, the largest trial ever done in anti-cytokine therapeutics, with an enrollment of 17,200 patients, will test the hypothesis whether an antibody to IL- $1 \beta$ will reduce cardiovascular events in a highrisk type 2 diabetes cohort with a CRP $>2.0 \mathrm{mg} / \mathrm{L}$ when on standard of therapy. In addition to blocking IL- $1 \beta$, clinical trials reveal that monoclonal antibodies to IL- $1 \alpha$ are also a therapeutic option in some diseases. IL- $1 \alpha$ is expressed on platelets and also present as an active precursor in most epithelial cells. Thus, IL- $1 \beta$ as well as IL- $1 \alpha$ emerge as master cytokines regulating diseases that were not appreciated as being due to endogenous inflammation.

\section{Conflict of Interest: None}

\section{References}

1 Dinarello CA. Interleukin-1 in the pathogenesis and treatment of inflammatory diseases. Blood 2011; 117: 3720-3732

2 Masters SL, Simon A, Aksentijevich I et al. Horror autoinflammaticus: the molecular pathophysiology of autoinflammatory disease. Annu Rev Immunol 2009; 27: 621 - 668

3 Hoffman HM, Mueller JL, Broide DH et al. Mutation of a new gene encoding a putative pyrin-like protein causes familial cold autoinflammatory syndrome and Muckle-Wells syndrome. Nat Genet 2001; 29: 301 - 305

4 Goldbach-Mansky R, Dailey NJ, Canna SW et al. Neonatal-onset multisystem inflammatory disease responsive to interleukin-1beta inhibition. N Engl J Med 2006; 355: 581 - 592

5 Reddy S, Jia S, Geoffrey R et al. An autoinflammatory disease due to homozygous deletion of the IL1RN locus. N Engl J Med 2009; 360: $2438-2444$

6 Aksentijevich I, Masters SL, Ferguson PJ et al. An autoinflammatory disease with deficiency of the interleukin-1-receptor antagonist. N Engl J Med 2009; 360: 2426-2437

\section{Bibliography}

Dol http://dx.doi.org/10.1055/s-0032-1324906

Arzneimittelforschung 2012; 62, Suppl. 1: S12-S12

(c) Georg Thieme Verlag KG Stuttgart · New York .

ISSN 0004-4172

Please note that the print version was published with a different, incorrect DOI. The correct DOI is the one shown here. 